\title{
Educação em Saúde promovendo prevenção de Infecções Sexualmente Transmissíveis em caminhoneiros através do uso de preservativos
}

\author{
Health Education promoting prevention of Sexually Transmitted Infections in truck drivers through \\ the use of condoms
}

\begin{abstract}
Educación en la salud que promueve la prevención de las infecciones de transmisión sexual en conductores de camiones mediante el uso de condons
\end{abstract}

Ana Carolina Bahia Perrone', Sérgio DonhaYarid², Tiago Ferraz Mascarenhas ${ }^{3}$

Como citar esse artigo. Perrone, ACB; Yarid, SD; Mascarenhas, TF. Educação em Saúde promovendo prevenção de Infecções Sexualmente Transmissíveis em caminhoneiros através do uso de preservativos. Revista Pró-UniverSUS. 2020 Jan./Jun.; 11 (1): 149-155.

\section{Resumo}

Os caminhoneiros, atualmente, compõem uma classe de trabalhadores expostos a diversos fatores que os colocam numa posição de vulnerabilidade. Ações de Educação em Saúde são propostas como tentativa de esclarecimento quanto ao uso de preservativos na prevenção das Infecções Sexualmente transmissíveis. Esse trabalho tem por objetivo promover educação em saúde relacionada ao uso de preservativos associado à prevalência de IST em caminhoneiros que trafegam na BR-116. Foi realizada uma ação de intervenção de educação em saúde multidisciplinar intitulada Saúde na BR, promovida por docentes e discentes do Núcleo de Pesquisa em Bioética e Espiritualidade da Universidade Estadual do Sudoeste da Bahia, que ocorre na base da Polícia Rodoviária Federal da BR-116 no município de Jequié, Bahia. Os 100 motoristas de caminhão de carga que participaram, eram na sua totalidade do sexo masculino. Destes, $90 \%$ possuíam vida sexual ativa e $10 \%$ já tiveram doença sexualmente transmissível. Somente $29 \%$ dos participantes responderam que sempre usam camisinha em suas relações sexuais, porém após as ações educativas, $60 \%$ deles passaram a afirmar que usarão sempre camisinha a partir daquele momento. Diante desses resultados, conclui-se a importância de medidas educativas na promoção da saúde assim como na prevenção de doenças infectocontagiosas.

Palavras-chave: Educação em Saúde, Caminhoneiros, IST, Preservativo.

\begin{abstract}
Nowadays, truck drivers make up a class of workers exposed to several factors that put them in a position of vulnerability. Health Education Actions are proposed as an attempt to clarify the use of condoms for the prevention of Sexually Transmitted Infections (STI). This study aims to promote health education related to the use of condoms associated with the prevalence of Sexually Transmitted Infections STI in truck drivers traveling on BR-116. A multidisciplinary health education intervention, named Health in BR, was promoted by teachers and students of the Núcleo de Pesquisa em Bioética e Espiritualidade da Universidade Estadual do Sudoeste da Bahia, and takes place at the base of the Highway Police Federal of BR-116 in the municipality of Jequié, Bahia. The 100 truck drivers who participated, were in their entirety male. Of these, $90 \%$ had an active sex life, and 10\% had Sexually Transmitted Disease (STD). Only $29 \%$ of the participants answered that they always use condoms in their sexual relations, but after the educational actions, $60 \%$ of them said that they will always use condoms from that moment. Because of these results, the importance of educational measures in the promotion of health, as well, as in the prevention of infectious diseases was concluded.
\end{abstract}

Keywords: Health Education, Truckers, STIs, Condom.

\section{Resumen}

Los camioneros de hoy constituyen una clase de trabajadores expuestos a varios factores que los colocan en una posición de vulnerabilidad. Las acciones de educación sanitaria se proponen como un intento de aclarar el uso de condones en la prevención de infecciones de transmisión sexual. Este estudio tiene como objetivo promover la educación para la salud relacionada con el uso del condón asociado con la prevalencia de ITS en conductores de camiones que transitan en la BR-116. Profesores y estudiantes del Centro de Investigación en Bioética y Espiritualidad de la Universidad Estatal del Sudoeste de Bahía, que ocurreen la base de la Policía Federal de Carreteras de BR-116, llevaron a cabo una acción de intervención de educación sanitaria multidisciplinaria titulada Salud en la BR, en el municipio de Jequié, Bahia. Los 100 conductores de camiones de carga que participaron eran todos hombres. De estos, el $90 \%$ tenía una vida sexual activa y el $10 \%$ tenía enfermedades de transmisión sexual. Solo el $29 \%$ de los participantes respondieron que siempre usan condones en sus relaciones sexuales, pero después de las acciones educativas, el $60 \%$ de ellos declararon que siempre usarán condones a partir de ese momento. Dados estos resultados, se concluye la importancia de las medidas educativas en la promoción de la salud, así como en la prevención de enfermedades infecciosas.

Palabras clave: Educación sobre camiones, Educación para la salud, IST, Condón.

Afiliação dos autores: 1. Professora da Universidade Estadual do Sudoeste da Bahia (UESB), Jequié, Bahia, Brasil.

2. Professor da Universidade Estadual do Sudoeste da Bahia (UESB), Jequié, Bahia, Brasil.

3. Acadêmico do curso de Medicina da Universidade Estadual do Sudoeste da Bahia (UESB), Jequié, Bahia, Brasil.

*Email de correspondencia: anabahiaperrone@hotmail.com 


\section{Introdução}

Muitos pesquisadores vêm desenvolvendo estudos que apontam os diversos contextos de vulnerabilidade vivenciados pelos caminhoneiros tanto no âmbito social quanto no individual ${ }^{1}$. O primeiro ponto a se destacar é que esses profissionais passam muitos dias fora de suas residências. Em estudo realizado por Teles et al. (2008), caminhoneiros que passam mais de 15 dias afastados de seus lares se relacionam mais com profissionais do sexo ${ }^{2}$. Em diversos estudos, o uso de preservativos nas relações sexuais é baixo, aumentando bastante o risco de contaminação para inúmeras doenças ${ }^{3,4,5}$. Outro fato importante é que muitos caminhoneiros relatam o uso de bebidas alcoólicas, drogas ilícitas e anfetaminas durante o ato sexual, com isso esquecem de usar proteção e consequentemente, aumentam ainda mais a sua vulnerabilidade ${ }^{2}$. O grau de escolaridade também se torna um fator determinante da saúde de uma população, haja vista que a maioria $(72 \%)$ dos motoristas de caminhão, no Brasil, não completam o Ensino Média ${ }^{6}$.

As Doenças Sexualmente Transmissíveis (DST) ou Infecções Sexualmente Transmissíveis (IST), termo mais usado na atualidade, são frequentes, possuem diversos agentes etiológicos envolvidos e várias apresentações clínicas. Elas causam impacto na qualidade de vida das pessoas, diminuem a fertilidade e interferem nas relações pessoais, familiares e sociais?. São infecções adquiridas através do ato sexual durante relação oral, vaginal ou anal, sendo representadas por: candidíase; cancromole; clamídia; condilomaacuminado; donovanose; gardnerella; gonorreia; hepatites A e B; herpes genital; HIV/Aids; linfogranuloma venéreo; sífilis e tricomoníase ${ }^{8}$. A Organização Mundial da Saúde (OMS) estima a ocorrência de mais de um milhão de IST por dia no mundo. Ao ano, esses números ultrapassam 357 milhões de novos casos de infecções entre clamídia, gonorreia, sífilis e tricomoníase ${ }^{9}$.

O uso da camisinha é o método mais barato, e acessível para a prevenção das DST, apesar de que, atualmente, reforça-se a ideia de que uma única estratégia de prevenção para as IST não é suficiente ${ }^{10}$.

A Educação em Saúde se caracteriza por um conjunto de ações de intervenção universais a vários grupos e campos de atuação, cujo propósito é sensibilizar o indivíduo e fazê-lo aderir às atividades propostas pelos educadores. Essas ações devem respeitar a disponibilidade de tempo, o local mais propício, os problemas apresentados e as características de cada grupo, mesmo tendo a possibilidade de serem realizadas em qualquer lugar ou em qualquer momento ${ }^{11}$. Essas práticas educativas precisam atingir seu público de forma a sensibilizá-los e mobilizá-los para que haja mudança de comportamento, não bastando somente passar bem o conteúdo, mas fazendo com que a informação gere uma ação ${ }^{3}$. Com isso, os objetivos da Educação em Saúde são de estimular, no indivíduo, o senso de responsabilidade pela sua própria saúde e pela saúde da comunidade em que estão inseridos, participando de maneira construtiva ${ }^{12}$.

Em outros países, como a Índia, onde o uso de preservativos entre os caminhoneiros que trafegam em Pune também é baixo, a conscientização sobre os métodos preventivos sobre o uso de preservativos contra a infecção pelo HIV é insuficiente, segundo estudos. Também nesse país com proporções continentais como o Brasil, há a necessidade de programas governamentais focados na mudança de comportamento, assim como programas educativos que incentivem a busca por atendimento médico e prevenção de doenças por parte dos motoristas de caminhão ${ }^{13}$.

Diante desse contexto de vulnerabilidade associada à falta de disponibilidade e acesso aos serviços de saúde, fica clara a necessidade de ações permanentes, por parte dos profissionais de saúde, que levem até os caminhoneiros algumas atividades educativas e de promoção à saúde. Com isso, esse trabalho se propõe a quantificar a prevalência de IST entre caminhoneiros atendidos em uma ação de intervenção em educação em saúde multidisciplinar e conferir a mudança de comportamento dos mesmos em relação ao uso de preservativos após exposição ao tema Infecções Sexualmente Transmissíveis.

\section{Materiais e Métodos}

Trata-se de um estudo exploratório, descritivo, transversal e de caráter quantitativo.

Foi adotada a metodologia quantitativa, associada a coleta de dados através de questionário semiestruturado, buscando analisar a relação entre a prevalência de IST entre caminhoneiros e o hábito do uso de preservativos. Após investigação, esses dados foram analisados estatisticamente.

Os participantes, ao chegarem no local, foram direcionados à recepção onde foram coletados os dados sociodemográficos através de uma ficha sem identificação do nome ou documento, assim como foi entregue o termo de consentimento livre e esclarecido (TCLE) para ser assinado antes do início da ação. Logo em seguida, foram entregues, a cada participante, todos os questionários referentes a todos os grupos disciplinares que avaliaram os caminhoneiros. Vale ressaltar que os motoristas foram orientados quanto a não obrigatoriedade das ações e participações em todos os estandes que foram divididos por profissões.

Ao chegarem no stand da medicina, os participantes receberam informações sobre infecções sexualmente transmissíveis e sobre a importância do uso 
de preservativos. Foram convidados a responderem o questionário e durante o preenchimento, foram mostradas fotos referentes às doenças sexualmente transmissíveis porque a última pergunta do questionário se refere à mudança de comportamento após a exposição do tema. Também foi distribuído um panfleto confeccionado pelo Ministério da Saúde referente ao tema, juntamente com preservativos.

A pesquisa foi realizada na Base da Polícia Rodoviária Federal, situada no Km 677 da BR-116 no município de Jequié, Bahia, no dia 21 de setembro de 2018 entre o intervalo de 6:00 horas da manhã até às 14:00 horas do mesmo dia.

O Saúde na BR consiste em uma ação de intervenção em Educação em Saúde multidisciplinar, realizado pelo Núcleo de Pesquisa em Bioética e Espiritualidade (NUB) do Programa de Pós-Graduação em Enfermagem e Saúde (PPGES) da Universidade Estadual do Sudoeste da Bahia - campus Jequié (UESB) em parceria com a Polícia Rodoviária Federal e a Via Bahia Concessionária de Rodovias S/A. Trata-se de um projeto de pesquisa e extensão realizado anualmente eque teve inicio no ano de 2014. Ele conta com a participação de estudantes de graduação dos cursos de Medicina, Odontologia, Farmácia, Educação Física, Enfermagem e Fisioterapia da UESB, estudantes de pós-graduação do PPGES, professores da Instituição e profissionais das áreas de Psicologia, Nutrição, Medicina, Enfermagem, Farmácia, Educação Física, Odontologia e Fisioterapia que participam do Núcleo de Pesquisa.

Esse projeto se propõe a promover saúde aos motoristas de caminhões que trafegam naBR-116, através de ações educativas e intervenções como aferição de pressão arterial, dosagem de glicemia capilar, avaliação física e antropométrica e promoção da saúde bucal com o objetivo de levar um pouco de conhecimento e bem estar para essa classe de trabalhadores que possuem grande dificuldade de buscarem as unidades de saúde devido à jornada de trabalho extenuante. Além disso, é oferecida uma refeição saudável com frutas diversas, sucos, pães, bolos, biscoitos, leite e café aos participantes sob a orientação de nutricionistas.

Foram participantes da pesquisa, exclusivamente condutores de caminhões que trafegam na estrada BR116 e que foram convidados pelos policiais rodoviários federais e aceitaram participar dessa ação. A amostra foi selecionada por método aleatório simples a partir da abordagem e convite realizados pelos policiais rodoviários federais

Foram excluídos deste estudo os acompanhantes dos caminhoneiros que estavam presentes no momento, porém que não são condutores, assim como os motoristas que se encontravam com algum distúrbio de comportamento.

A pesquisa foi realizada respeitando as diretrizes da Resolução 466/12 do Conselho Nacional de Saúde
(CNS), que aborda os aspectos éticos da pesquisa envolvendo seres humanos pautada nos princípios básicos da autonomia, beneficência, bioética, equidade, não maleficência e justiça.

As pessoas que aceitaram participar assinaram o Termo de Consentimento Livre e Esclarecido (TCLE), autorizando a coleta, além de cederem os direitos de uso e divulgação do conteúdo, bem como autorização para publicação dos resultados da pesquisa em artigos, revistas e divulgação em eventos técnico-científicos nacionais e internacionais. Foi garantido ao participante o sigilo e anonimato além de assegurar-lhe o direito de sua desistência em qualquer momento da pesquisa.

\section{Resultados e Discussão}

No presente estudo, foram preenchidas 117 fichas na recepção, porém no stand da medicina participaram 100 caminhoneiros devido ao fato de 3 entrevistados na recepção serem acompanhantes e 11 se recusarem a preencher o questionário proposto. Ainda houve 3 desistências.

Os dados a seguir referem-se à situação sociodemográfica dos participantes: Todos os entrevistados (100\%) foram do sexo masculino, condizendo com a Confederação Nacional dos Transportes - CNT que demonstra que 99,8\% dos motoristas de caminhão de carga no Brasil são homens. Esse número inexpressivo de mulheres dirigindo caminhões no Brasil é reflexo de uma sociedade machista, que ainda não igualou os espaços laborais para os dois gêneros. Além disso, os pontos de parada para caminhoneiros nas estradas não possuem estrutura adequada para as mulheres, faltando, muitas, vezes até mesmo banheiros destinados para essa classe. A falta de segurança nas estradas também é um fator desestimulante, assim como a necessidade de força física para descarregar as cargas, e principalmente, a maternidade priva a mulher de exercer a profissão, tanto no período gestacional quanto no puerpério e lactação obrigando-as a permanecerem muitos meses afastadas do trabalho se optarem por terem filhos ${ }^{6}$.

A idade variou de 21 a 69 anos, sendo que 6 deles possuem mais de 60 anos, compatível com o envelhecimento e aumento da expectativa de vida da população. Quanto ao grau de escolaridade, somente 2 deles possuem nível superior, contrastando com 39 que possuem nível fundamental e 44 que possuem nível médio. Quinze não responderam. Esses resultados são equivalentes aos da pesquisa realizada por Krause e Carniel (2014) com caminhoneiros que demonstrou uma faixa etária entre 18 a 66 anos e uma porcentagem de $25 \%$ que cursaram até o ensino fundamental e $3 \%$ que cursaram o ensino superior. Esse perfil de baixa escolaridade pode impactar diretamente nos maus 
hábitos de vida dos caminhoneiros, assim como para a manutenção de algumas práticas que interferem diretamente na saúde e qualidade de vida como a falta de atividade física, baixa ingesta de alimentos ricos em fibras, dentre outras ${ }^{14}$. Além disso, a baixa escolaridade favorece ao uso de drogas ilícitas, substâncias para se manterem acordados e ingesta de álcool, aumentando a vulnerabilidade desses trabalhadores ${ }^{15}$.

A jornada de trabalho diária declarada pelos participantes variou entre 5 e 24 horas, sendo a média igual a 10,27 horas, estando de acordo com a média encontrada por Kapron (2012) igual a 13 horas diárias e 6,1 dias por semana. No referido estudo, o número

Figura 1. Relação do Estado Civil dos Caminhoneiros entrevistados.

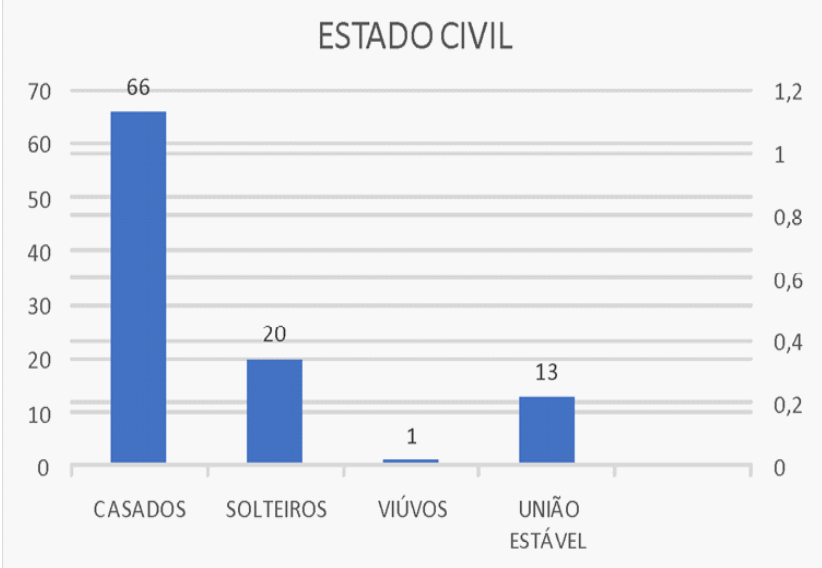

Fonte: Dados da Pesquisa. elevado de horas trabalhadas por dia está diretamente relacionado ao número alto de acidentes de trabalho onde $27 \%$ dos acidentes de trânsito ocorridos em rodovias e estradas no ano de 2000 foram provocados por caminhões ${ }^{16}$.

Os próximos dados são referentes ao questionário aplicado no stand da medicina cujos gráficos, a seguir, mostram o estado civil dos motoristas e se eles possuem vida sexual ativa, assim como se costumam ter relações com mais de uma parceira por ano. Eles apontam que somente $20 \%$ dos entrevistados são solteiros, $90 \%$ possuem vida sexual ativa e $78 \%$ declaram possuírem apenas 1 parceira sexual.

Figura 2. Porcentagem dos Caminhoneiros que possuem vida sexual ativa.

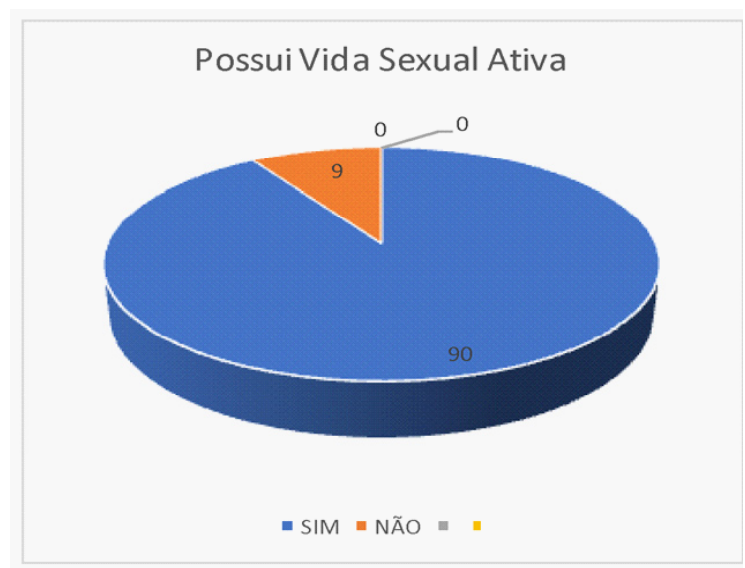

Fonte: Dados da Pesquisa

Gráfico 03: Número médio de parceiras sexuais por ano.

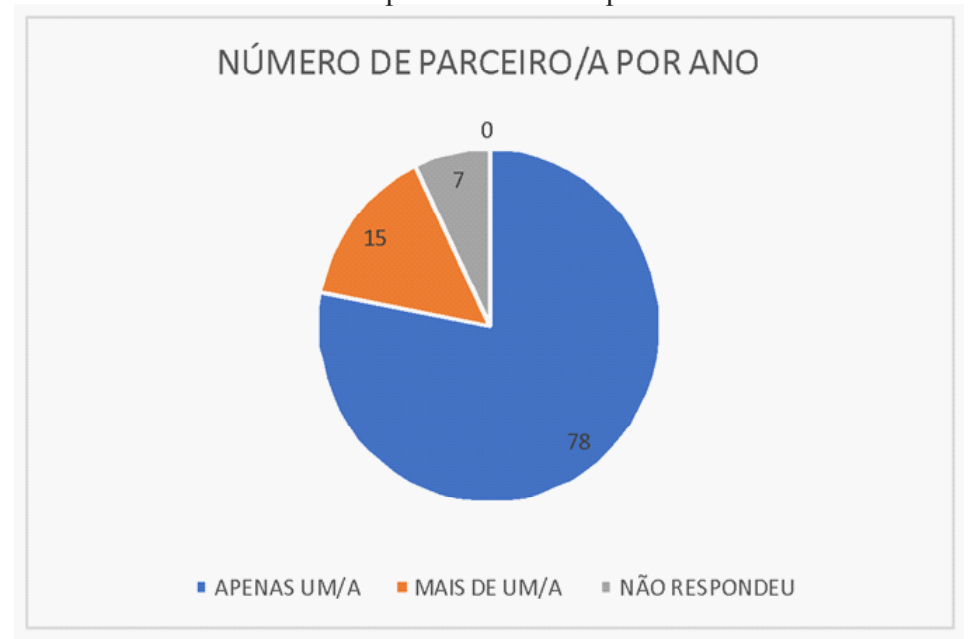

Fonte: Dados da Pesquisa 
No estudo de Teles et al. (2008), onde 77,7\% dos caminhoneiros entrevistados eram casados e $15,4 \%$ eram solteiros e ainda que a prevalência de DST foi diretamente associada à prática de relação sexual sem preservativo com parceiras fixas e também com relação sexual com profissionais do sexo, foi demonstrado que o fato de ser casado não é fator protetor contra as IST. Fica claro também que quanto maior o número de parceiros/ as eles tiverem, maior será a chance de exposição e contaminação ${ }^{2}$.

Quando perguntado se eles sabiam o que é uma Doença Sexualmente Transmissível, responderam que $\operatorname{sim} 88 \%$ deles e $12 \%$ responderam que não. Esse valor de $12 \%$, apesar de ser aparentemente baixo, significa que algumas pessoas ainda não possuem conhecimento sobre os riscos de adquirirem alguns tipos de doenças e isso provavelmente está diretamente associado ao baixo grau de escolaridade. Esse fato é preocupante porque, consequentemente, os caminhoneiros desconhecem os métodos de prevenção das DST, sendo mais necessárias ainda atividades educativas de promoção à saúde capazes de suprir essa falta de conhecimento ${ }^{17}$.

Dentre os 100 caminhoneiros entrevistados no presente estudo $90 \%$ declararam nunca terem tido alguma Infecção Sexualmente Transmissível, porém
$10 \%$ afirmaram que já tiveram, sendo 3 por Herpes genital, 5 por Gonorreia e 2 se negaram a responder qual foi a etiologia da doença. Essa pergunta foi respondida após eles terem visto fotos de algumas DST, por isso, alguns deles podem não saber que tiveram tais doenças e por isso não responderam já que estudos mostram que a prevalência dessas doenças é bem maior no Brasil. Magalhães et al. (2015), coletaram amostra de sangue de 152 caminhoneiros para realização de teste rápido para Hepatites B e C e demonstrou uma prevalência das DST em 31\% deles, de Hepatite B em 14\% e Hepatite C em 7\%. Com isso, o autor constata que esses profissionais, por possuírem grande mobilidade geográfica, atuam como disseminadores de infecções, incluindo as transmitidas por ato sexual ${ }^{18}$. Em outro estudo, realizado por Barbosa et al. (2015), onde foram realizados teste rápidos para HIV 1 e 2 e Sífilis em 666 caminhoneiros, foram descobertos $6(0,9 \%)$ deles com teste positivo para HIV e $69(10,4 \%)$ motoristas com teste rápido positivo para Sífilis. Nele concluiu-se que um dos principais fatores envolvidos nessa prevalência foi o tempo de permanência fora de seus lares que reflete a carência afetiva por falta da família ${ }^{19}$.

O gráfico a seguir mostra os resultados das perguntas: Você usa camisinha?

Figura 4. Porcentagem dos Caminhoneiros que usam camisinha.

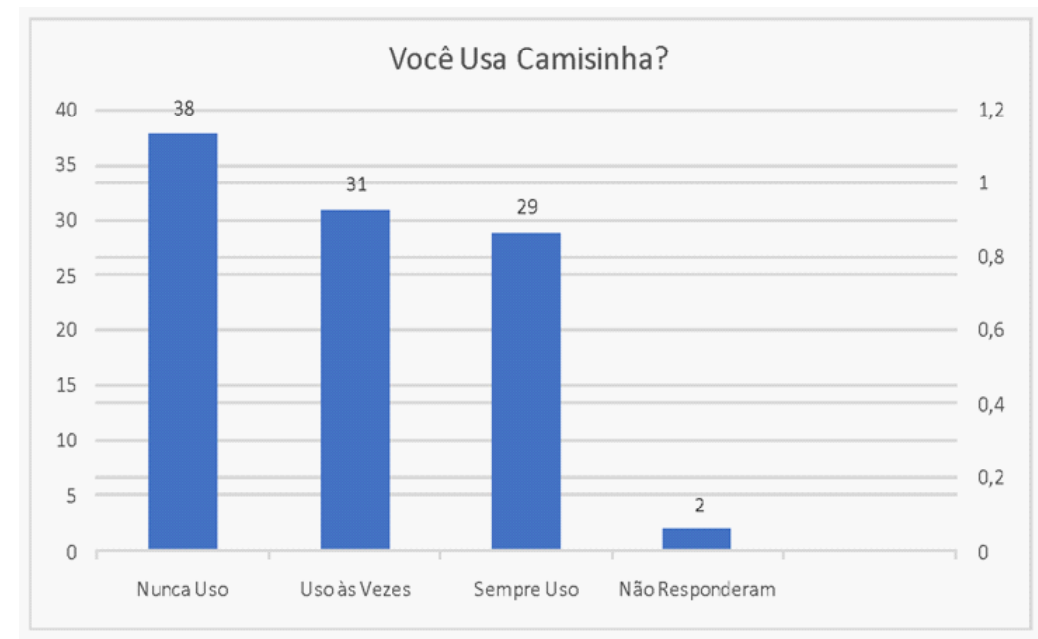

Fonte: Dados da Pesquisa

Percebe-se, nessa pergunta, que somente $29 \%$ dos entrevistados afirmaram que sempre usam camisinha nas relações sexuais. A relação entre o uso de preservativos e a incidência de doenças transmitidas pelo ato sexual está intimamente relacionada e é abordada com clareza no estudo de Codeset al. (2006) onde somente 37,6\% dos entrevistados faziam uso do preservativo enquanto $21,3 \%$ deles já haviam adquirido alguma DST. Esse estudo também revela que os homens procuram menos os serviços de saúde, possuem mais parceiras sexuais e são mais disseminadores dessas doenças quando comparados às mulheres, mais uma vez colocando os caminhoneiros em posição de protagonistas do seu próprio comportamento de risco $^{20}$. Madureira e Trentini (2008) realizaram um estudo sobre o uso de preservativos entre casais heterossexuais e alencou diversas causas para a baixa adesão ao preservativo entre esses casais como a diminuição do prazer pelo homem perante a redução do contato físico com a mulher; a desconfiança por parte da mulher de que o homem pode estar tendo outros relacionamentos extraconjugais; a perda de uma oportunidade de uma relação inesperada 
ou não programada, dentre outras, reservando o hábito de usar camisinha somente para prevenir uma gravidez indesejada $^{21}$.

Por fim, após todas as explicações e exposições de fotos e materiais explicativos, foi perguntado se os entrevistados passariam a usar o preservativo após aquele dia, obtendo como resultados: $20 \%$ responderam continuarei sem usar; $17 \%$ responderam tentarei lembrar de usar; 3\% não responderam e $60 \%$ responderam usarei sempre. Quando comparamos os $60 \%$ que responderam que usarão sempre o preservativo com a pergunta anterior onde somente $29 \%$ deles usavam sempre a camisinha, constatamos que as medidas educativas aumentaram mais de $50 \%$ as intenções de se aderir ao uso da proteção no ato sexual, comprovando que as ações educativas são eficazes na promoção da saúde. Benzakenet al. (2007) realizou um trabalho de intervenção com 500 mulheres trabalhadoras do sexo e publicou que $94,7 \%$ das participantes mudaram seu comportamento sexual para prevenirem as DST após as ações de intervenção. Ele ainda pontua que a utilização dessas estratégias educativas podem contribuir para a transformação individual e também de uma população, garantindo, não apenas resultados sociopolíticos que interfiram na vulnerabilidade às IST, assim como garantir o direito à saúde ${ }^{22}$.

\section{Considerações Finais}

No atual estudo, foi possível perceber o quanto as atividades de intervenção através da Educação em Saúde são importantes e eficazes. Os caminhoneiros, atualmente, fazem parte de um grupo de profissionais que movimentam a economia do país, porém as políticas de saúde existentes, como a Saúde do Trabalhador, por exemplo, não conseguem incluir essa classe nos seus propósitos. A jornada de trabalho dos motoristas de caminhões é o principal fator de exclusão deles dos atendimentos de saúde.

$\mathrm{E}$ enquanto os caminhoneiros permanecem às margens das ações de saúde promovidas no nosso país, se transformam em potenciais disseminadores de doenças infectocontagiosas, não somente para suas famílias, assim como para toda a população em todo território nacional, devido à grande mobilidade deles.

Através de ações simples como o ato de levar informações a respeito de determinadas doenças e como preveni-las, garantimos a oportunidade de promoção da saúde, não só para esses trabalhadores, assim como para toda a sociedade. Por isso, investir em Educação em Saúde se faz necessário para garantirmos os Direitos Humanos de nossa população.

\section{Referências bibliográficas}

1. Sousa LMS, Silva LS, Palmeira AT. Representações sociais de caminhoneiros de rota curta sobre HIV/AIDS. Psicologia \& Sociedade, 2014; 26(2):346-355.

2. Teles AS, Matos MS, Caetano KAA, Costa LA, França DDS, Pessini GC, Brunini SM, Martins RMB. Comportamentos de risco para doenças sexualmente transmissíveis em caminhoneiros no Brasil. Rev Panam Salud Publica, 2008; 24(1):25-30.

3. Villarinho L, Bezerra I, Lacerda R, Latorre MRDO, Paiva V, Stall R, Hearst N. Caminhoneiros de rota curta e sua vulnerabilidade ao HIV, Santos, SP. Rev Saúde Pública, 2002; 36(4) Supl:61-7.

4. Fry PH, Monteiro S, Maio MC, Bastos FI, Santos RV. AIDS tem cor ou raça? Interpretação de dados e formulação de políticas de saúde no Brasil. Cad. Saúde Pública. Rio de Janeiro, 2007; 23(3):497-523.

5. Araújo VG, Botelho EC, Amadei JL. Medidas de Prevenção para DST/AIDS relatadas por Caminhoneiros do Norte do Paraná.Iniciação Científica CESUMAR,2012; 14(1):63-70.

6. Pesquisa CNT. Perfil dos Caminhoneiros. Brasília: CNT, 2019. $132 \mathrm{p}$.

7. Brasil. Manual de Bolso das Doenças Sexualmente Transmissíveis. Ministério da Saúde, Secretaria de Vigilância em Saúde, Programa Nacional de DST e AIDS. Brasília: Ministério da Saúde. Série Manuais n. 24. 2. ed. 2005. 108p.

8. Rocha EM. DST e Aids em região de fronteiras:um estudo com caminhoneiros no Estado de Rondônia. [dissertação]. Cacoal (RO): Universidade de Brasília; 2008.

9. Organización Mundial de La Salud. Orientacionesmundiales sobre loscriterios y procesos para lavalidación de laeliminación de latransmisión materno infantil del VIH y la sífilis. Ginebra: OMS, 2015.

10. Dourado I, MacCarthy S, Reddy M, Calazans G, Gruskin S. Revisitando o uso do preservativo no Brasil. RevBrasEpidemiol, 2015; 18 suppl.1:63-88.

11. Sobrinho-Santos CK, Silva AV, Malheiros AF, Trindade RA, Pagan AA. Relatos de caminhoneiros sobre a prevenção do HIV e o material educacional impresso: reflexões para educação em saúde. Ciênc. Educ. Bauru. 2015; 21(4):1011-1030. DOI: http://dx.doi.org/10.1590/1516731320150040014

12. Oliveira HM, Gonçalves MJF. EDUCAÇÃO EM SAÚDE: uma experiência transformadora. RevBrasEnferm. Brasília (DF), 2004; 57(6):761-3.

13. Yadav AK, Gupta H, Vaz LS, Yadav J. Human immunodeficiency virus, sexually transmitted disease awareness and condom usage among long-distance internal truck drivers in Pune, India. HIV AIDS Rev, 2018; 17(1):40-48. DOI: https://doi.org/10.5114/hivar. 2018.73978.

14. Krause C, Carniel F. Sono, Estado Nutricional e Hábitos de Vida de Caminhoneiros que trafegam pela BR 364. Revista Científica da Faculdade de Educação e Meio Ambiente,2014; 5(2):125-138.

15. Knauth DR, Pilecco FB, Leal AF, Seffner F, Teixeira AMFB. Manterse acordado: a vulnerabilidade dos caminhoneiros no Rio Grande do Sul. Rev Saúde Pública, 2012; 46(5):886-93.

16. Kapron RA. Tempo, jornada e produtividade na história e trabalho dos caminhoneiros. Revista Latino-Americana de História,2012; 1(3):194206.

17. Masson VA, Monteiro MI. Vulnerabilidade à Doenças Sexualmente Transmissíveis/AIDS e uso de drogas psicoativas por caminhoneiros. RevBrasEnferm, Brasília, 2010; 63(1):79-83.

18. Magalhães HJC, Carvalho ACM, Silva THS, Monteiro-Neto V, Monteiro AS, Bomfim MRQ, Monteiro SG. Comportamentos de risco para a infecção pelos vírus da hepatite B em caminhoneiros de longa distância em São Luís-MA. Rev. Investig, Bioméd., São Luís, 2015; 7:35-46. DOI: https:// doi.org/10.24863/rib.v7i1.18.

19. Barbosa RMG, Diniz SP, Costa CDD, Rodrigues DS, Alcântara KC. Soroprevalência Para HIV e Sífilis em Caminhoneiros que trafegam pela BR 
153 no Centro-Oeste Brasileiro. Revista de Biotecnologia \& Ciência, 2015; $4(1)$.

20. Codes JS, Cohen DA, Melo NA, Teixeira GG, Leal AS, Silva TJ, Oliveira MPR. Detecção de doenças sexualmente transmissíveis em ambientes clínicos e não clínicos na Cidade de Salvador, Bahia, Brasil. Cad. Saúde Pública. Rio de Janeiro, 2006; 22(2):325-334.

21. Madureira VSF, Trentini M. Da utilização do preservativo masculino à prevenção de DST/AIDS. Ciência \& Saúde Coletiva, 2008; 13(6):18071816.

22. Benzaken AS, Garcia EG, Sardinha JCG, Pedrosa VL, Paiva V. Intervenção de base comunitária para a prevenção das DST/AIDS na região amazônica, Brasil. Rev Saúde Pública, 2007; 41(Supl.2):118-26. 